\title{
A Simple Set of Test Matrices for Eigenvalue Programs*
}

\author{
By C. W. Gear**
}

Abstract. Sets of simple matrices of order $N$ are given, together with all of their eigenvalues and right eigenvectors, and simple rules for generating their inverses in the nonsingular cases. In general, these matrices are nonsymmetric. They can have sets of double and triple roots. In each of these cases, two of the roots of the doublet or triplet can correspond to a single eigenvector.

The general form of the $N \times N$ matrix is:

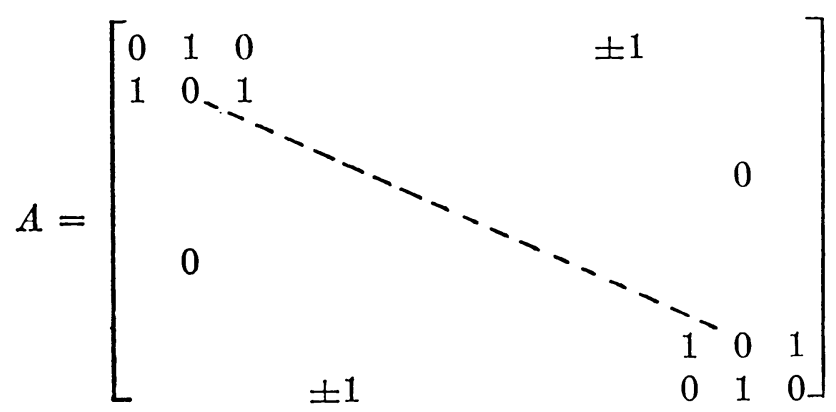

where the \pm 1 is in the $J$ th column of the first row and in the $(N+1-K)$ th column of the last row. The first row can be expressed as

$$
e_{2}{ }^{T} \pm e_{J}{ }^{T},
$$

where $e_{i}$ is the vector consisting of a unit in the $i$ th position. The special case in which the first row is simply $e_{2}{ }^{T}$ will be shown to be equivalent to taking $J=0$ with a negative sign in (1). (Notationally this does not make sense, but it will save writing to include it in this way.) The last row can be similarly written as $e_{N-1}^{T} \pm e_{N+1-K}^{T}$. Note that $J$ and $K$ and the signs may be different.

The eigenvalues and eigenvectors of these matrices may all be expressed in the form:

$$
\text { Eigenvalue }=2 \cos \alpha .
$$

Right eigenvector $\xi=[\sin (\omega+\alpha), \sin (\omega+2 \alpha), \cdots, \sin (\omega+N \alpha)]^{T}$.

The values of $\alpha$ and $\omega$ are given below. In this discussion, all roman letters represent integers, sets, or matrices.

Define the sets of $\alpha$ and $\omega$ values by Tables I and II.

The eigenvalues and eigenvectors are then the union of the sets of $\alpha$ and $\omega$ values specified in Table III. This union gives all $N$ eigenvalues and as many eigenvectors as exist.

Received April 25, 1968, revised June 27, 1968.

* Work supported in part by the U. S. Atomic Energy Commission under grant AEC AT(11-1) 1469 and by the Argonne National Laboratory.

** Department of Computer Science, University of Illinois, Urbana, Illinois. 
TABLE I. Sets of $\alpha$ Values

\begin{tabular}{clc}
\hline Set Name & $\alpha$ Members \\
\hline$A 1$ & $2 p \pi /(2 N+2-J-K)$ & $1 \leqq p<N+1-(K+J) / 2$ \\
\hline$A 2$ & $(2 p-1) \pi /(2 N+2-J-K) 1 \leqq p \leqq N+1-(K+J) / 2$ \\
\hline$B 1$ & $2 p \pi / J$ & $1 \leqq p<J / 2$ \\
\hline$B 2$ & $(2 p-1) \pi / J$ & $1 \leqq p \leqq J / 2$ \\
\hline$C 1$ & $2 p \pi / K$ & $1 \leqq p<K / 2$ \\
\hline$C 2$ & $(2 p-1) \pi / K$ & $1 \leqq p \leqq K / 2$ \\
\hline$D$ & 0 & \\
\hline$E$ & $\pi$
\end{tabular}

TABLE II. Sets of $\omega$ Values

\begin{tabular}{|c|c|c|}
\hline Set Name & $\omega$ Members & \\
\hline$X 1$ & $(\pi-J \alpha) / 2$ & These first four sets \\
\hline$X 2$ & $-J \alpha / 2$ & are generated by \\
\hline$Y 1$ & $(\pi-(2 N+2-K) \alpha) / 2$ & corresponding sets \\
\hline$Y 2$ & $-(2 N+2-K) \alpha / 2$ & of $\alpha$ values. \\
\hline$Z$ & $\pi / 2$ & \\
\hline
\end{tabular}

Proofs. The proof of each statement is trivial but tedious; therefore only the double positive case will be explored in detail.

The $i$ th element $\xi_{i}$ of the eigenvector $\xi$ is $\sin (\omega+i \alpha)$. The $i$ th element of $A \xi$ is:

$$
\begin{aligned}
\sin (\omega+(i-1) \alpha) & +\sin (\omega+(i+1) \alpha), \quad \text { if } 1<i<N, \\
= & 2 \cos \alpha \sin (\omega+i \alpha), \\
& =2 \xi_{i} \cos \alpha .
\end{aligned}
$$

The first element of $A \xi$ is

$$
\sin (\omega+2 \alpha) \pm \sin (\omega+J \alpha) .
$$

We would like this to be equal to $2 \xi_{i} \cos \alpha$. Note that

$$
\sin \omega+\sin (\omega+2 \alpha)=2 \xi_{i} \cos \alpha
$$

so that we want

$$
\sin \alpha= \pm \sin (\omega+J \alpha) .
$$

Similarly, we want 


$$
\sin (\omega+(N+1) \alpha)= \pm \sin (\omega+(N+1-K) \alpha) .
$$

(2) and (3) form a pair of equations which determine $\alpha$ and $\omega$ such that $2 \cos \alpha$ and $\xi$ are an eigenvalue and eigenvector of $A$.

TABLE III. Eigenvalues

\begin{tabular}{|c|c|c|c|c|}
\hline \multicolumn{2}{|c|}{$\begin{array}{l}\text { Sign of extra } \\
\text { one digit in }\end{array}$} & \multirow[t]{2}{*}{$\alpha$ Values } & \multirow[t]{2}{*}{$\begin{array}{l}\text { Corresponding } \\
\omega \text { Values }\end{array}$} & \\
\hline row 1 & row $N$ & & & \\
\hline \multirow[t]{4}{*}{+} & + & $A 1$ & $X 1$ & Note 1 \\
\hline & & $B 1$ & $\begin{array}{l}Y 1 \\
X 1\end{array}$ & Note 2 \\
\hline & & $D$ & $\hat{Z}^{1}$ & \\
\hline & & $E$ if $K, J$ both even & $Z$ & Note 3 \\
\hline \multirow[t]{3}{*}{+} & - & $A 2$ & $X 1$ & Note 1 \\
\hline & & $\begin{array}{l}B 1 \\
C 2\end{array}$ & $\begin{array}{l}Y 2 \\
X 1\end{array}$ & Note 2 \\
\hline & & $E$ if $K$ odd, $J$ even & $Z$ & Note 3 \\
\hline \multirow[t]{3}{*}{-} & + & $\begin{array}{l}A 2 \\
B 2\end{array}$ & $\begin{array}{l}X 2 \\
Y 1\end{array}$ & Note 1 \\
\hline & & $C 1$ & $X 2$ & Note 2 \\
\hline & & $E$ if $K$ even, $J$ odd & $Z$ & Note 3 \\
\hline \multirow[t]{3}{*}{-} & - & $A 1$ & $X 2$ & Note 1 \\
\hline & & $B 2$ & $\begin{array}{l}Y 2 \\
Y 2\end{array}$ & Note? \\
\hline & & $E$ if $K, J$ both odd & $\begin{array}{l}X 2 \\
Z\end{array}$ & $\begin{array}{l}\text { Note } 2 \\
\text { Note } 3\end{array}$ \\
\hline
\end{tabular}

Note 1: If $A$ and $B$ or $A$ and $C$ sets have a nonnull intersection, each $\alpha$ in the intersection corresponds to a double root $\lambda$ with a single eigenvector $\xi$. A solution of $(A-\lambda I) \mu=\xi$ is given by: $\mu=(-1 / 2 \sin \alpha)[\cos (\omega+\alpha), 2 \cos (\omega+\alpha), \cdots, N \cos (\omega+N \alpha)]^{T}$.

Note 2: If the $B$ and $C$ sets have a nonnull intersection, each $\alpha$ in the intersection corresponds to a double root with two eigenvalues. The second can be found by adding $\pi / 2$ to the $\omega$ given in the table.

Note 3: If $J$ and $K$ do not satisfy the condition, set $E$ is not included.

Note that if the first row is simply $e_{2}{ }^{T}$, then $\sin \omega=0$ replaces Eq. (2). This is identical to requiring that (2) hold with a minus sign and $J=0$. We will proceed by solving (2) and (3) and showing that all $N$ eigenvalues have been accounted for. either

Consider only the case with plus signs in (2) and (3). Equation (2) implies that

$$
\omega+2 p \pi=\omega+J \alpha,
$$

or

$$
\omega=(2 p+1) \pi-(\omega+J \alpha),
$$

while Eq. (3) implies that either

$$
\omega+(N+1) \alpha+2 q \pi=\omega+(N+1-K) \alpha,
$$




$$
\omega+(N+1) \alpha=(2 q \pi+1)-(\omega+(N+1-K) \alpha) .
$$

One of each of these pairs must be used.

(4.1) and (5.1) imply that:

$$
\alpha=2 p \pi / J=2 q \pi / K, \quad \omega \text { arbitrary . }
$$

These cases will usually be included in other cases, but two special cases should be noted now. If $p=q=0, \alpha=0$. $\omega$ can be chosen as $\pi / 2$. This gives set $D$ which only occurs in the ++ case. The eigenvector is $[1,1,1, \cdots, 1]^{T}$.

If $J, K$ are both even, $p=J / 2, q=K / 2$ gives $\alpha=\pi$. Again, $\omega$ can be chosen as $\pi / 2$. This gives $\alpha$ set $E$. The eigenvector is $[1,-1,1,-1, \cdots]^{T}$.

(4.2) and (5.2) imply that

$$
2 \omega=(2 p+1) \pi-J \alpha=(2 q+1) \pi-(2 N+2-K) \alpha .
$$

Let $q=0$; since $p$ is arbitrary, then

$$
\alpha=2 p \pi /(2 N+2-J-K) .
$$

$p=0$ has already been handled. $p \geqq(2 N+2-J-K) / 2$ has been handled (equality case) or is equivalent to smaller $p$. ( $\alpha$ should remain in the range $[0, \pi]$. ) Therefore $1 \leqq p<(2 N+2-J-K) / 2$ and this case gives $\alpha$ set $A 1$. From (7) we get $\omega$ set $X 1$ since multiples of $\pi$ may be discarded in $\omega$.

(4.1) and (5.2) imply that

$$
\begin{aligned}
\alpha & =2 p \pi / J, \\
2 \omega & =(2 q+1) \pi-(2 N+2-K) \alpha .
\end{aligned}
$$

Again we can take $1 \leqq p<J / 2$ and $q=0$ to get $\alpha$ set $B 1$ and $\omega$ set $Y 1$. Similarly (4.2) and (5.1) lead to $\alpha$ set $C 1$ and $\omega$ set $X 1$.

We now have a set of values and vectors. The remaining problem is to show that there are no others. First note that we have $N$ values in Table III. In the ++ case, for example, we have sets $A 1, B 1, C 1, D$ and $E$ if $J$ and $K$ are even. This gives a total of

$[(2 N+2-K-J-1) / 2]+[(J-1) / 2]+[(K-1) / 2]+1$

( +1 if $J, K$ both even) .

For each of the four cases we have

\begin{tabular}{ccc}
\hline$J$ & $K$ & Number of Members \\
\hline odd & odd & $N+1-(K+J) / 2-1+(J-1) / 2+(K-1) / 2+1=N$ \\
\hline odd & even & $N+1-(K+J) / 2-\frac{1}{2}+(J-1) / 2+(K-2) / 2+1=N$ \\
\hline even & odd & $N+1-(K+J) / 2-\frac{1}{2}+(J-2) / 2+(K-1) / 2+1=N$ \\
\hline even & even & $N+1-(K+J) / 2-1+(J-2) / 2+(K-2) / 2+1+1=N$ \\
\hline
\end{tabular}

Therefore, if the $\alpha$ sets do not intersect, we have all of the values and vectors. If, for example, sets $A 1$ and $B 1$ have a nonempty intersection, we have: 


$$
\alpha=2 p_{1} \pi /(2 N+2-J-K)=2 p_{2} \pi / J,
$$

with

$$
1 \leqq p_{1}<N+1-(J+K) / 2, \quad 1 \leqq p_{2}<J / 2 .
$$

This implies that $J$ and $(2 N+2-J-K)$ have a common factor of at least 3 . In this case consider

$$
\nu=(A-2 \cos \alpha I) \mu
$$

where

If $1<i<N$,

$$
\mu_{i}=-i \cos (\omega+i \alpha) / 2 \sin \alpha .
$$

$$
\begin{aligned}
-2 \nu_{i} \sin \alpha= & (i-1) \cos (\omega+(i-1) \alpha)-2 i \cos \alpha \cos (\omega+i \alpha) \\
& +(i+1) \cos (\omega+(i+1) \alpha), \\
= & i[\cos (\omega(i-1) \alpha)+\cos (\omega+(i+1) \alpha)-2 \cos \alpha \cos (\omega+i \alpha)] \\
& +\cos (\omega+(i+1) \alpha)-\cos (\omega+(i-1) \alpha), \\
= & -2 \sin \alpha \sin (\omega+i \alpha), \\
= & -2 \sin \alpha \xi_{i} .
\end{aligned}
$$

Therefore,

$$
\nu_{i}=\xi_{i}
$$

For $i=1$, we have

$$
-2 \nu_{1} \sin \alpha=2 \cos (\omega+2 \alpha)-2 \cos \alpha \cos (\omega+\alpha)+J \cos (\omega+J \alpha) .
$$

From (11), $J \alpha=2 p_{2} \pi$, and in this case the $\omega$ value can be obtained from the set $X 1$. Therefore,

$$
\begin{aligned}
\cos (\omega+J \alpha) & =\cos ((\pi-J \alpha) / 2+J \alpha)=\cos ((\pi+J \alpha) / 2) \\
& =\cos \left(\pi+2 p_{2} \pi\right) / 2=0,
\end{aligned}
$$

which implies that $\nu_{1}=\xi_{1}$. Similarly, we can show that $\nu_{N}=\xi_{N}$. Thus, if sets $A 1$ and $B 1$ intersect, we get a double root $\lambda$ with a single vector $\xi$ and a solution of $(A-\lambda I) \mu$ $=\xi$ as given above.

A similar result holds for intersections of $A 1$ and $C 1$. If $B 1$ and $C 1$ intersect, then $\alpha=2 p_{1} \pi / J=2 p_{2} \pi / K$. This corresponds to Eq. (6), which means that $\omega$ is arbitrary. By taking the $\omega$ given in Table II and $\pi / 2$ plus that $\omega$, two linearly independent eigenvectors are obtained. If $A 1, B 1$, and $C 1$ have a common intersection, then a triple root with two eigenvectors and one other principal vector will be obtained. Therefore, we have obtained a complete set of eigenvalues.

Example. $N=8, K=J=6$, double positive case.

Matrix

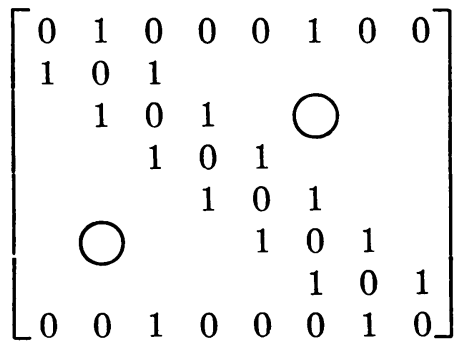


$\alpha$ Values

Set $A 1 \quad \pi / 3 \quad 2 \pi / 3$

Set $B 1 \quad \pi / 3 \quad 2 \pi / 3$

Set $C 1 \quad \pi / 3 \quad 2 \pi / 3$

Set $D \quad 0$

Set $E \quad \pi$ $\omega$ Values

$-\pi / 2 \quad-3 \pi / 2$

$-\pi / 2 \quad-3 \pi / 2$

$-\pi \quad-2 \pi$

$\pi / 2$

$\pi / 2$
Eigenvalues

$-1, \quad 1$

$-1, \quad 1$

$-1, \quad 1$

2

$-2$

The inverses of a set of related matrices, $2 I-A$, are easy to compute. (These matrices can occur in one space dimension boundary value problems. Their eigenvalues are $2(1-\cos \alpha)$.) The Ith column of the inverse has the form

$$
\frac{1}{a-b}[w+a, w+2 a, \cdots, w+I a, w+I a+b, \cdots, w+I a+(N-I) b]^{T}
$$

if $2 \leqq I \leqq N-1$, and

$$
[w+a, w+2 a, \cdots, w+N a]^{T}
$$

if $I=1$ or $N$, where the $w, a$ and $b$ must be chosen (separately for each $I$ ) to satisfy equations arising from the first and last rows of the matrix. ( $w, a$ and $b$ may be noninteger). Thus, for $N=6, I=2, J=5$ in the -+ case, the matrix and its inverse are

$$
\left[\begin{array}{rrrrrr}
2 & -1 & 1 & 0 & 0 & 0 \\
-1 & 2 & -1 & 0 & 0 & 0 \\
0 & -1 & 2 & -1 & 0 & 0 \\
0 & 0 & -1 & 2 & -1 & 0 \\
0 & 0 & 0 & -1 & 2 & -1 \\
0 & -1 & 0 & 0 & -1 & 2
\end{array}\right] \text { and } \frac{1}{20}\left[\begin{array}{rrrrrr}
10 & 0 & -8 & -6 & -4 & -2 \\
10 & 20 & 8 & 6 & 4 & 2 \\
10 & 20 & 24 & 18 & 12 & 6 \\
10 & 20 & 20 & 30 & 20 & 10 \\
10 & 20 & 16 & 22 & 28 & 14 \\
10 & 20 & 12 & 14 & 16 & 18
\end{array}\right]
$$

respectively.

These results can be extended to cover the types of matrices arising from differential equations in two or more space dimensions. We will treat the extension from one to two dimensions. Consider the block matrix

$$
B=\left[\begin{array}{rrrrrrr}
A & D & & & \pm D & & \\
D & A & D & & & & \\
& D & A & D & & & \\
& & & & D & A & D \\
& & \pm D & & & D & A
\end{array}\right] .
$$

Where the matrix $A$ is as defined earlier, the matrix $D$ is a diagonal matrix with elements $\delta$ and where the extra $\pm D$ blocks appear in the $J^{\prime}$ th and $N^{\prime}+1-K$ th block columns, or do not appear at all. Consider the vector

$$
\nu=\left[\mu_{1} \xi, \mu_{2} \xi, \cdots, \mu_{N}^{\prime} \xi\right]^{T},
$$

where $\xi$ is an eigenvector of $A$ corresponding to $\lambda$. We will write this as $\mu \times \xi$.

The $i$ th block of $B \nu$ for $1<i<N^{\prime}$ is

$$
D \mu_{i-1} \xi+A \mu_{i} \xi+D \mu_{i+1} \xi=\left(\delta\left(\mu_{i-1}+\mu_{i+1}\right) / \mu_{i}+\lambda\right) \mu_{i} \xi .
$$

Hence, if the first and last blocks can be fixed appropriately, and if 


$$
\delta\left(\mu_{i-1}+\mu_{i+1}\right) / \mu_{i}+\lambda=\lambda^{\prime}, \quad 1<i<N^{\prime},
$$

$\nu$ will be an eigenvector of $B$ corresponding to $\lambda^{\prime}$.

(13) is a recurrence relation for $\mu_{i}$ with a solution

$$
\mu_{i}=\sin \left(\omega^{\prime}+i \alpha^{\prime}\right),
$$

when $\lambda^{\prime}=2 \xi \cos \alpha^{\prime}+\lambda$. The first block of $B \nu$ leads to the requirement that

$$
\mu_{0}= \pm \mu_{J^{\prime}}
$$

while the last block requires that

$$
\mu_{N^{\prime}+1}= \pm \mu_{N^{\prime}+1-K^{\prime}} .
$$

(15) and (16) determine $\omega^{\prime}$ and $\alpha^{\prime}$ by use of Tables I, II, and III, so that the eigenvalues and eigenvectors of $B$ can be determined from (12).

In the case of repeated roots without a complete set of eigenvectors, the principal vectors can also be used for $\mu$ or $\xi$. They will give rise to principal vectors of $B$. If $\mu$ and $\xi$ are the single eigenvectors associated with double roots $\alpha^{\prime}$ and $\alpha$ respectively, and if $\bar{\mu}$ and $\bar{\xi}$ are the associated principal vectors of order 2 , then it is trivial to show that $\mu \times \bar{\xi}, \bar{\mu} \times \bar{\xi}$ are principal vectors of orders 2 and 3 , while $\bar{\mu} \times \xi-\mu \times \bar{\xi}$ and $\mu \times \xi$ are independent eigenvectors.

The extension to any number of dimensions is straightforward. The use of more dimensions allows multiple roots to be introduced. For example, the $5 \times 5$ matrix

$$
A=\left[\begin{array}{lllll}
0 & 1 & 0 & 1 & 0 \\
1 & 0 & 1 & 0 & 0 \\
0 & 1 & 0 & 1 & 0 \\
0 & 0 & 1 & 0 & 1 \\
0 & 1 & 0 & 1 & 0
\end{array}\right]
$$

has roots $-2,0,0,0$ and 2 . If the process above is applied twice, we get a matrix of order 125 with at least 27 zero roots and principal vectors of order 4 .

The author would like to acknowledge the helpful comments of Professor Fosdick and the referee. 